\title{
An Adaptive Cascaded ILA- and DLA-Based Digital Predistorter for Linearizing an RF Power Amplifier
}

\author{
Han Le Duc , Bruno Feuvrie, Matthieu Pastore, and Yide Wang
}

\begin{abstract}
This paper presents a novel adaptive digital predistortion (DPD) technique based on a cascade of an adaptive indirect learning architecture (ILA) and a static direct learning architecture (DLA) using a linear interpolation look-up-table (LILUT). The static LILUT-DLA-based DPD is designed to identify the inverse of a radio-frequency power amplifier (PA) model. The cascaded system of the DLA-based predistorter (PD) and PA is theoretically linear. However, in real-time applications, the PA characteristics change with time due to process, supply voltage, and temperature variations, making this cascaded system not strictly linear, which results in some residual nonlinear distortion at the PA output. This residual distortion is effectively compensated by an additional adaptive ILA-based PD using least mean squares or recursive least squares. Thanks to the incorporation of the static DLA, the proposed DPD approach is less sensitive to the PA output noise, ensuring a better preinverse of the PA and also requiring a smaller number of adaptive coefficients than either the adaptive stand-alone DLA- or ILA-based DPDs. The experimental results show that the proposed DPD technique effectively linearizes the PA, even if its characteristics change, and obtains better linearization performance than either the classical stand-alone DLA- or stand-alone ILA-based DPDs.
\end{abstract}

Index Terms-Nonlinear systems, power amplifier, adaptive digital predistortion (DPD), indirect learning architecture (ILA), direct learning architecture (DLA), adaptive algorithm.

\section{INTRODUCTION}

$\mathbf{M}$ ODERN communication systems are continuously evolving to satisfy the requirement of high data rate for multimedia communications. The signals of these systems have high peak-to-average power ratio (PAPR) and wide bandwidth, leading to stringent linearity requirements for signal amplification.

Manuscript received March 27, 2018; revised July 24, 2018 and September 18, 2018; accepted September 21, 2018. This work was supported by BPI France, region Occitanie and region Nouvelle Aquitaine, through the APOGEES project. This paper was recommended by Associate Editor J. Lota. (Corresponding author: Han Le Duc.)

H. Le Duc is with the Institute of Electronics and Telecommunications of Rennes, UMR CNRS 6164, University of Nantes, 44035 Nantes, France, and also with Telerad Company, 64603 Anglet, France (e-mail: h.leduc@telerad.fr).

B. Feuvrie and Y. Wang are with the Institute of Electronics and Telecommunications of Rennes, UMR CNRS 6164, University of Nantes, 44035 Nantes, France.

M. Pastore is with Telerad Company, 64603 Anglet, France.

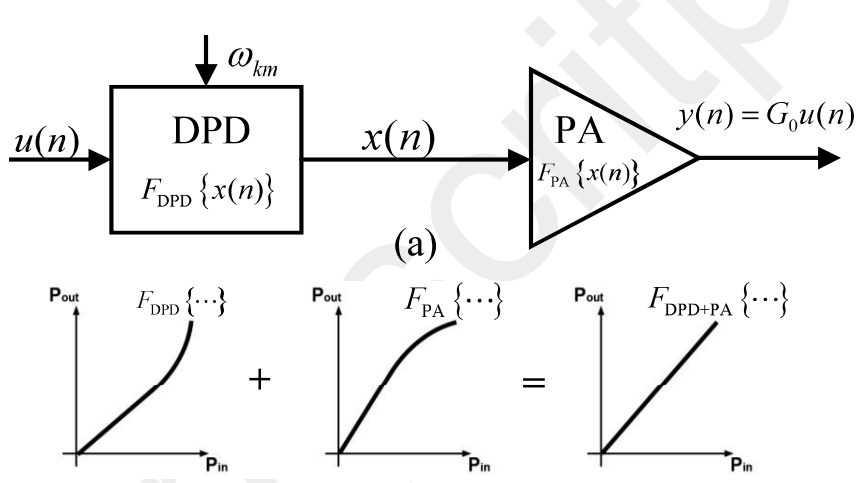

(b)

Fig. 1. DPD concept. (a) Block diagram. (b) Transfer functions of DPD unit, $\mathrm{PA}$, and DPD+PA system.

Two key quality factors of radio frequency (RF) power amplifiers (PAs) in modern wireless communication systems are efficiency and linearity. Unfortunately, it is hard to simultaneously obtain the above two requirements due to the inherent characteristics of PAs. In order to maximize the efficiency, PAs should operate close to saturation, producing strong nonlinear distortion in the amplified signal. This distortion increases the error vector magnitude (EVM) and also results in spectral regrowth, causing interference to neighboring channels and increasing the adjacent channel power ratio (ACPR). By operating the PA far below saturation, its nonlinear behavior can be reduced. However, this leads to low power efficiency because of the high PAPR of modern communication signals. As a result, the PA design has to make a tradeoff between efficiency and linearity. In order to fulfill the efficiency requirement without sacrificing linearity, PA linearization techniques are required [1]. Thanks to its highly cost-effective and easy implementation, baseband digital predistortion (DPD) is a popular and widely used linearizion technique [2]-[11]. Its principle is shown in Fig. 1. The DPD concept places a predistorter (PD) block in front of the PA. Ideally, the nonlinear transfer function of the PD is the inverse of that of the PA. Consequently, the cascaded PD and PA system becomes linear and the original input is amplified by a constant gain. The PD is added in the baseband, working entirely in the digital domain. The performance of the DPD is controlled by a set of complex coefficients $\omega_{k m}$ that can be estimated either offline, based on a block of measured input and output samples of 


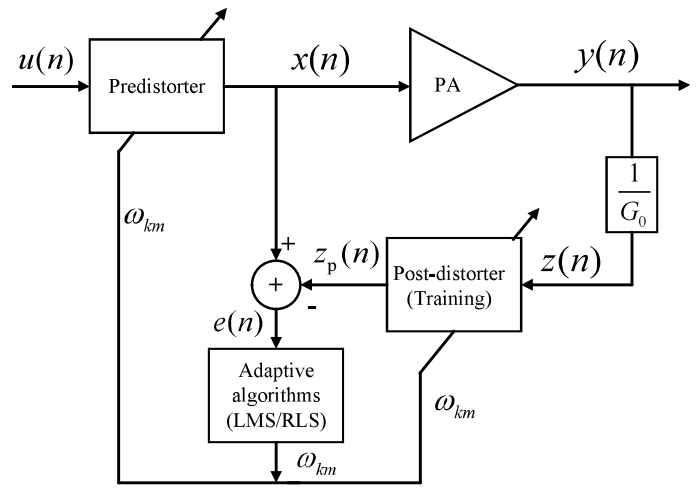

Fig. 2. Block diagram of conventional ILA-based DPD.

the PA, or online in an adaptive way based on real-time measurements of the PA input and output signals [9], [12]. Since the characteristics of the PA are usually unknown, learning architectures and adaptive algorithms are employed to estimate its inverse function. There are two commonly used learning architectures for identifying the parameters of a PD: indirect learning architecture (ILA) [12]-[17] and direct learning architecture (DLA) [8]-[11], [18]-[20]. The ILA-based DPD computes an inverse model of the PA via the post-distorter (or training) block, whose input is the output of the PA as illustrated in Fig. 2, where $G_{0}$ is the gain of the linearized PA. The PD transfer function is an exact duplicate of that of the training block. At convergence, the identified coefficients of the post-distorter are copied to the PD, making the cascaded PD and PA system behave linearly. The ILA-based DPD architecture can be performed either in an offline manner using least squares (LS) [3] or in an adaptive manner using an adaptive algorithm such as least mean squares (LMS) or recursive least squares (RLS) [4], [21]. The key advantage of the ILAbased DPD is its implementation simplicity [15]. However, it suffers from two typical drawbacks [12], [16], [17]. Firstly, the adaptive algorithm may converge to a biased solution due to the presence of noise in the measured PA output. Secondly, the efficiency of the ILA-based PD performance is poor when the PA works near its saturation region [4], [17]. To cope with the ILA-based PD noise-induced bias problem, the output of a forward model of the PA can be used instead of using the real noisy PA output to identify the inverse model [14], [16]. Although these approaches yield better linearity performance than the original ILA-based PD, their performance depends on the accuracy of the PA forward model [4].

The above mentioned drawbacks are not present in DLA-based DPD techniques, which can be performed either online [9]-[11], as shown in Fig. 3(a), or offline [8], [22], as illustrated in Fig. 3(b). In the adaptive case, the PD parameters are identified by comparing the wanted signal $G_{0} u(n)$ with the PA output $y(n)$. The error produced at the PA output is minimized by using an adaptive algorithm such as LMS or RLS [10], [12], [14], [17], [21]. For these approaches, the computation of an instantaneous estimate of the gradient of mean square error with respect to the PD coefficients is complex and computationally expensive. Thus, most of

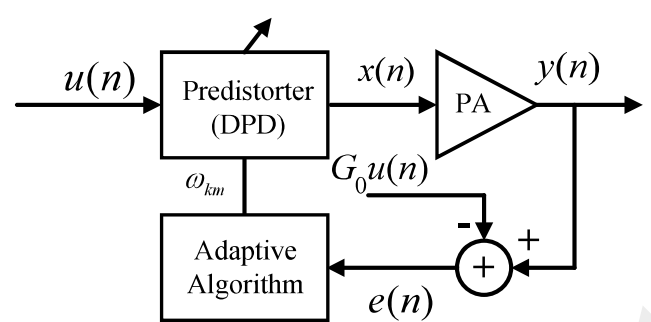

(a)

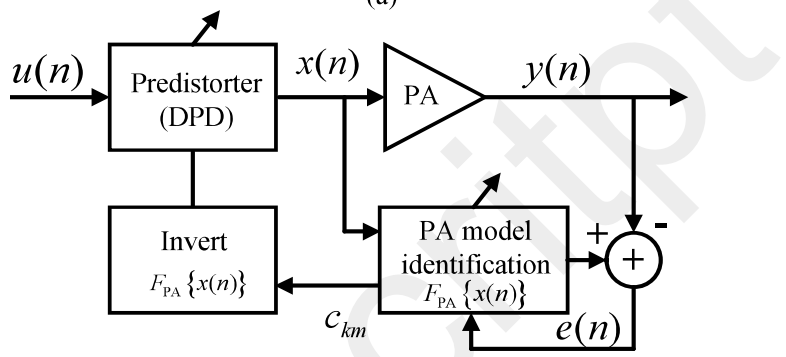

(b)

Fig. 3. Block diagram of DLA-based DPD. (a) Online. (b) Offline.

these are complex in structure, and also suffer from slow convergence [4]. In the offline case, the coefficients of the PA model are extracted using the LS method after gathering a set of input and output samples of the PA. The PD identification techniques proposed in [8], [22], and [23] use a look-uptable (LUT) to significantly reduce the computational time required in a conventional memory-polynomial-based DPD. However, the crucial drawback of these DLA-based DPD techniques is to require a sufficiently large LUT in order to obtain good linearization performance. To cope with this drawback, the DLA-based linearization technique in [8] proposes a linearly interpolated LUT (LILUT) algorithm, increasing the indexing efficiency of the LUT, and hence reducing the LUT size. This is actually an improved solution of the LUT-DLA-based DPD proposed in [22]. Although the LILUTDLA-based DPD proposed in [8] shows good performance with low complexity and fast convergence, it is limited to applications where the PAs are operating under relatively stable conditions, e.g., the PA characteristics remain almost constant over time. However, in practice, the PA characteristics may change rapidly with time due to process, supply voltage, and temperature (PVT) variations. If the static DPD in [8] is employed, the different DPD functions or coefficients must be readapted to changes in the PA characteristics, which is more difficult than an ILA-based DPD.

Considering the aforementioned pros and cons of conventional learning architectures (DLA and ILA) and the offline LILUT-DLA-based DPD proposed in [8], we propose in this paper, a novel adaptive DPD architecture cascading the adaptive ILA-based PD and offline (or static) LILUT-DLAbased PD. The static LILUT-DLA-based PD is designed to linearize the PA for a specific condition such that the cascaded system of the DLA-based PD and PA, named as CDPA in the rest of the paper, is theoretically linear. As the PA characteristics change with time due to PVT variations, the CDPA is no longer linear, causing some residual nonlinear 
distortion at the PA output. Since the most significant part of the PA nonlinear memory effects is compensated by the static DLA-based PD, this residual distortion can be effectively mitigated by the proposed adaptive ILA-based PD placed in front of the CDPA. Thanks to the incorporation of the static DLA-based PD with the PA, the CDPA is much less nonlinear than the PA and the proposed additional adaptive ILA-based $\mathrm{PD}$ will be less complex and easier to design than either the adaptive stand-alone DLA- or ILA-based PDs. As a result, the proposed DPD solves several problems that arise when using either the stand-alone DLA- or ILA-based DPDs to linearize a PA whose characteristics change due to PVT drifts.

The rest of the paper is organized as follows. Section II reviews the offline (or static) stand-alone LILUT-DLA-based DPD. Section III describes the proposed adaptive linearization technique. Experimental validation results are presented in Section IV. Conclusions are drawn in Section V.

\section{Review of the OfFline StAnd-Alone LILUT-DLA-BASED DPD}

This section reviews the offline (or static) stand-alone DLA-based DPD using the LILUT proposed in [8]. A simple block diagram is shown in Fig. 3(b). Due to its simple implementation, the MP-based model is widely applied for behavioral modeling and predistortion of PAs and transmitters exhibiting nonlinear memory effects [1], [2].

Let $c_{k m}$ denote the coefficients of the MP-based model of a PA. These coefficients can be identified by the LS technique using the input and output data measured from the PA as in [24]. The input and output of the PA model can be expressed as [3]

$$
y(n)=\sum_{k=1}^{N} \sum_{m=0}^{M} c_{k m} x(n-m)|x(n-m)|^{k-1},
$$

where $N$ and $M$ are, respectively, the nonlinear order and memory depth of the MP-based model of the PA, and $y(n)$ and $x(n)$ denote, respectively, the output and input samples of the PA. Ideally, the output of the CDPA system is

$$
y(n)=G_{0} u(n),
$$

where $u(n)$ denotes the input of the CDPA system. The output $y(n)$ can be decomposed into two parts: the static part $s(n)$ depending only on the current input sample $(m=0)$ and the dynamic part $d(n)$ formed by only the previous input samples $(m=1, \cdots, M)$ as

$$
y(n)=s(n)+d(n)=G_{0} u(n),
$$

where

$$
s(n)=\sum_{k=1}^{N} c_{k 0} x(n)|x(n)|^{k-1},
$$

and

$$
d(n)=\sum_{k=1}^{N} \sum_{m=1}^{M} c_{k m} x(n-m)|x(n-m)|^{k-1},
$$

TABLE I

INPUT AND OUTPUT OF LILUT

\begin{tabular}{l|c|c}
\hline \multirow{2}{*}{ LILUT Input } & \multicolumn{2}{|c}{ LILUT Output } \\
\cline { 2 - 3 } & Amplitude $R(k)$ & Slope for amplitude $S_{R}(k)$ \\
\hline$E(0)$ & $R(0)$ & $S_{R}(0)$ \\
$\cdots$ & $\cdots$ & $\cdots$ \\
$E(k)$ & $R(k)$ & $S_{R}(k)$ \\
$\cdots$ & $\cdots$ & $\cdots$ \\
$E(K-1)$ & $R(K-1)$ & $S_{R}(K-1)$ \\
$E(K)$ & $R(K)$ & $\times$ \\
\hline
\end{tabular}

where $|x(n)|$ denotes the amplitude of the predistorted signal $x(n)$. Given linear gain $G_{0}$ and the baseband input $u(n)$, the goal of identification is to determine the predistorted signal $x(n)$ at the PD output such that the CDPA system behaves linearly. To solve this optimization problem, (4) is rewritten as

$$
s(n)=e^{j \alpha} \sum_{k=1}^{N} c_{k 0}|x(n)|^{k}=G_{0} u(n)-d(n),
$$

where $\alpha$ is the phase of the predistorted signal $x(n)$. The rightmost side of (6) can be computed at time instant $n$. Taking absolute value of both sides of (6), the amplitude of the predistorted signal is then the real positive root (which always exists [25]) of the following polynomial:

$$
\left.\left|\sum_{k=1}^{N} c_{k 0}\right| x(n)\right|^{k}|-| G_{0} u(n)-d(n) \mid=0 .
$$

The roots of the polynomial of the left-hand side of (7) can be determined by a classical root-finding process [25].

Although the root-finding process shows good linearization performance, it is very time-consuming. Thus, it is not applicable in a real-time application. Therefore, in [8], the root-finding process is substituted by a LILUT algorithm, which estimates the amplitude $|x(n)|$ and phase $\alpha$ of $x(n)$ based on (6). Firstly, the dynamic range of $|x(n)|$ is estimated according to the characteristics of the PA [26]. Secondly, the determined dynamic range of $|x(n)|$ is divided into $K$ intervals with equal length $|\Delta x|$. The LILUT is then constructed and shown in Table I, where its input $E(k)$ is computed as

$$
E(k)=\left.\left|\sum_{i=1}^{N} c_{i 0}\right| k \Delta x\right|^{i} \mid,
$$

and its two outputs, corresponding to the amplitude $R(k)$ and amplitude slope $S_{R}(k)$, are calculated as

$$
\begin{aligned}
R(k) & =k \Delta x, \\
S_{R}(k) & =\frac{R(k+1)-R(k)}{E(k+1)-E(k)}, \quad 0 \leq k \leq K-1 .
\end{aligned}
$$

Finally, the amplitude and phase of the predistorted signal $x(n)$ are determined by Algorithm 1, where $L$ is the number of training samples. 


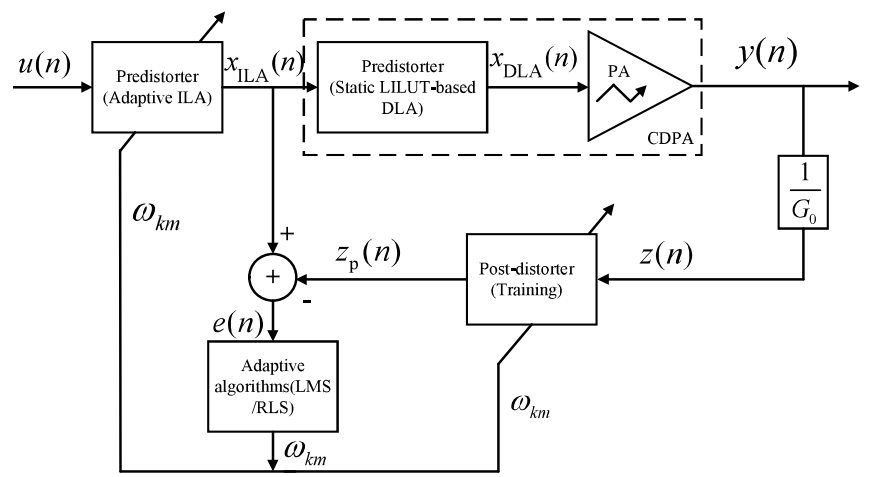

Fig. 4. Proposed adaptive DPD using a combined learning architecture.

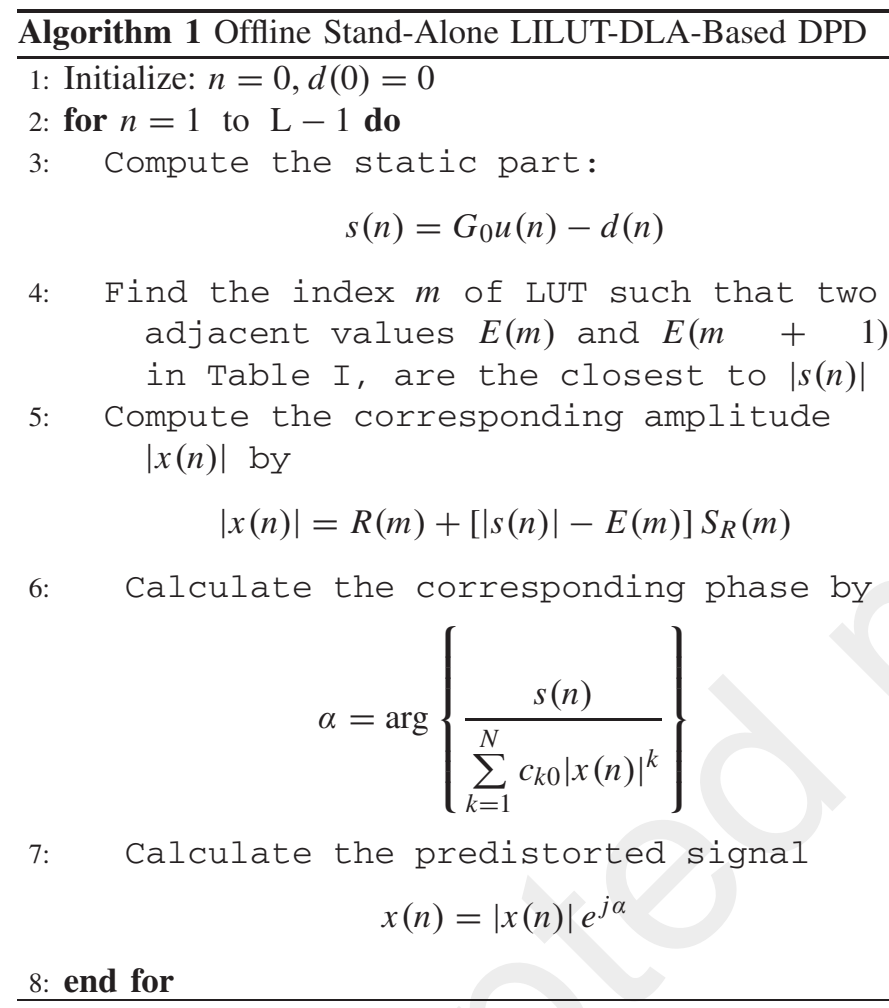

\section{Proposed Online DPD Architecture}

The offline LILUT-DLA-based DPD presented in Section II is limited to applications where the PA characteristics do not change with time. However, this scenario is not the case in practice, especially in reconfigurable systems where the signal type, modulation, power, etc., can change completely. Furthermore, the adaptive stand-alone ILA-based DPD has a noise problem.

When the PA works close to its saturation region, the correlation matrix used to determine the inverse model of the PA is badly conditioned. As a result, the identified model parameters are very sensitive to noise at the PA output. Thus, we propose a novel adaptive DPD technique shown in Fig. 4, where a combined DLA and ILA architecture is designed. The proposed architecture consists of an adaptive ILA-based PD followed by a static LILUT-DLA-based PD. The static LILUT-DLA-based PD uses the LILUT algorithm presented in Algorithm 1, where a static LILUT in Table I is first computed for the PA working under a specific condition (temperature). The static CDPA system shown in Fig. 4 is theoretically linear if the PA characteristics do not change. However, if the PA characteristics change, the CDPA is no longer linear, resulting in some residual nonlinear distortion at the PA output. The proposed additional ILA-based PD using LMS or RLS can effectively mitigate this residual nonlinear distortion. Thanks to the incorporation of the static DLA-based PD with the PA, the correlation matrix of the CDPA output samples is better conditioned than that of the uncompensated PA output samples. Thus, the proposed DPD is more robust to noise at the PA output than the stand-alone ILA-based DPD, and can efficiently track coefficient fluctuations of the PA due to PVT drift.

\section{A. Cascaded Architecture of the LMS-ILA-Based PD and Static LILUT-DLA-Based PD}

The standard ILA-based DPD proposed in [3], [12], [13], [21], and [27] is applied in our solution, shown in Fig. 4. The postinverse of the CDPA is identified using a post-distorter (or training) block, where $z(n)$ and $z_{\mathrm{p}}(n)$ are the input and output of the post-distorter, respectively. The PD transfer function is an exact duplicate of that of the training block. The ILA-based PD has input $u(n)$ and output $x_{\mathrm{ILA}}(n)$.

We assume that both the ILA-based PD and post-distorter are modeled by a MP. The MP-based model of the ILA-based $\mathrm{PD}$ is expressed as [3], [23]

$$
x_{\mathrm{ILA}}(n)=\sum_{k=1}^{Q} \sum_{m=0}^{P} \omega_{k m} u(n-m)|u(n-m)|^{k-1},
$$

where $Q$ and $P$ are the nonlinear order and memory depth of the MP-based model, respectively, and $\omega_{\mathrm{km}}$ are the coefficients of the model. The input and output of the post-distorter are expressed as

$$
z_{\mathrm{p}}(n)=\sum_{k=1}^{Q} \sum_{m=0}^{P} \omega_{k m} z(n-m)|z(n-m)|^{k-1},
$$

with

$$
z(n)=\frac{y(n)}{G_{0}} .
$$

Let us define a new sequence as

$$
z_{k m}(n)=z(n-m)|z(n-m)|^{k-1},
$$

In matrix form, the output of the post-distorter (12), can be expressed as

$$
z_{\mathrm{p}}(n)=\mathbf{z}^{\mathrm{T}}(n) \omega,
$$

where symbol $\mathrm{T}$ indicates matrix transpose and

$$
\mathbf{z}(n)=\left[z_{10}(n), \ldots, z_{Q 0}(n), \ldots, z_{1 P}(n), \ldots, z_{Q P}\right]^{\mathrm{T}} .
$$

The coefficient vector $\omega$ is denoted by

$$
\omega=\left[\omega_{10}, \ldots, \omega_{Q 0}, \ldots, \omega_{1 P}, \ldots, \omega_{Q P}\right]^{\mathrm{T}} .
$$

The error signal is defined by

$$
e(n)=x_{\mathrm{ILA}}(n)-z_{\mathrm{p}}(n)=x_{\mathrm{ILA}}(n)-\mathbf{z}^{\mathrm{T}}(n) \omega .
$$


The LMS algorithm minimizes the mean square error $E\left\{|e(n)|^{2}\right\}$ to identify the coefficients $\omega_{k m}$. The updating equation is expressed as [28], [29]

$$
\begin{aligned}
\hat{\omega}(n) & =\hat{\omega}(n-1)-\frac{1}{2} \mu \frac{\partial|e(n)|^{2}}{\partial \hat{\omega}(n)} \\
& =\hat{\omega}(n-1)+\mu e(n) \mathbf{z}(n),
\end{aligned}
$$

where $\mu$ is the step-size parameter of the LMS technique. Finally, the proposed adaptive DPD technique is described in Algorithm 2 where $F_{\mathrm{PA}}\{\cdots\}$ is the PA transfer function, modeled by a MP function.

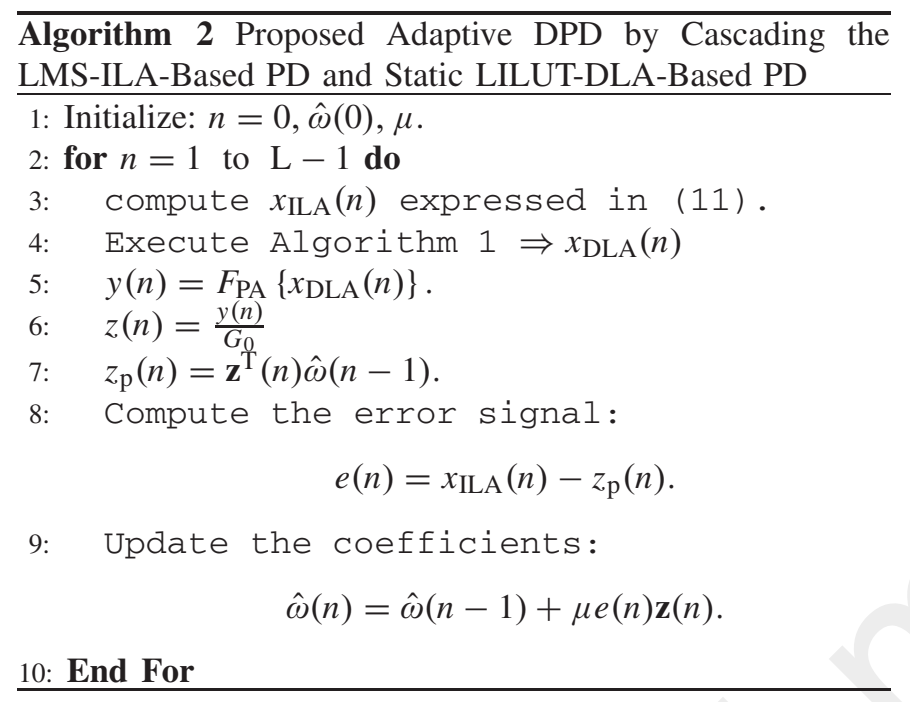

The key advantage of the LMS algorithm is its low computational complexity. The price paid for this simplicity is slow convergence since LMS uses only the step-size to govern its convergence speed and steady-state misadjustment. Thus, it is hard to make an optimal trade-off between them [29].

\section{B. Cascaded Architecture of the RLS-ILA-Based PD and Static LILUT-DLA-Based PD}

In order to obtain faster convergence, the RLS algorithm [28], [29] is applied in our framework, to minimize the following weighted sum of magnitude-squared errors:

$$
\zeta=\sum_{l=0}^{n} \lambda^{n-l}|e(l)|^{2},
$$

where $0<\lambda<1$ is the "forgetting factor". An adaptive cascaded architecture of ILA and DLA using RLS is shown in Algorithm 3, where $\delta$ is a positive scalar determined by experimentation in order to balance the stability with the convergence rate. Typically, $\delta>100 \sigma_{x}^{2}$, where $\sigma_{x}^{2}$ is the variance of the input. The value of $\lambda$ is commonly chosen in the range $0.95<\lambda<1$ [28], [29].

To simplify the notation, the proposed adaptive DPD architecture of the cascaded ILA- and DLA-based PDs using LMS is denoted as Design $+L M S$, and likewise using RLS as Design $+R L S$.
Algorithm 3 Proposed Adaptive DPD by Cascading the RLS-ILA-Based PD and Static LILUT-DLA-Based PD

$$
\begin{aligned}
& \text { 1: Initialize: } n=0, \hat{\omega}(0), \mathbf{P}_{0}=\delta \mathbf{I} \\
& \text { 2: for } n=1 \text { to } \mathrm{L}-1 \text { do } \\
& \text { 3: compute } x_{\mathrm{ILA}}(n) \text { expressed in }(11) . \\
& \text { 4: } \quad \text { Execute Algorithm } 1 \Rightarrow x_{\mathrm{DLA}}(n) \\
& \text { 5: } y(n)=F_{\mathrm{PA}}\left\{x_{\mathrm{DLA}}(n)\right\} . \\
& \text { 6: } \quad z(n)=\frac{y(n)}{G_{0}} \\
& \text { 7: } \quad z_{\mathrm{p}}(n)=\mathbf{z}^{\mathrm{T}}(n) \hat{\omega}(n-1) . \\
& \text { 8: } \quad \text { Compute the error signal: } \\
& \qquad e(n)=x_{\mathrm{ILA}}(n)-z_{\mathrm{p}}(n) .
\end{aligned}
$$

9: Compute the Kalman gain vector:

$$
\mathbf{k}(n)=\frac{\mathbf{P}(n-1) \mathbf{z}(n)}{\lambda+\mathbf{z}^{\mathrm{T}}(n) \mathbf{P}(n-1) \mathbf{z}(n)}
$$

10: Update the inverse of the correlation matrix:

$$
\mathbf{P}(n)=\frac{1}{\lambda}\left[\mathbf{P}(n-1)-\mathbf{k}(n) \mathbf{z}^{\mathrm{T}}(n) \mathbf{P}(n-1)\right]
$$

11: 5. Update the coefficients of the post-distorter:

$$
\hat{\omega}(n)=\hat{\omega}(n-1)+\mathbf{k}(n) e(n)
$$

\section{2: End For}

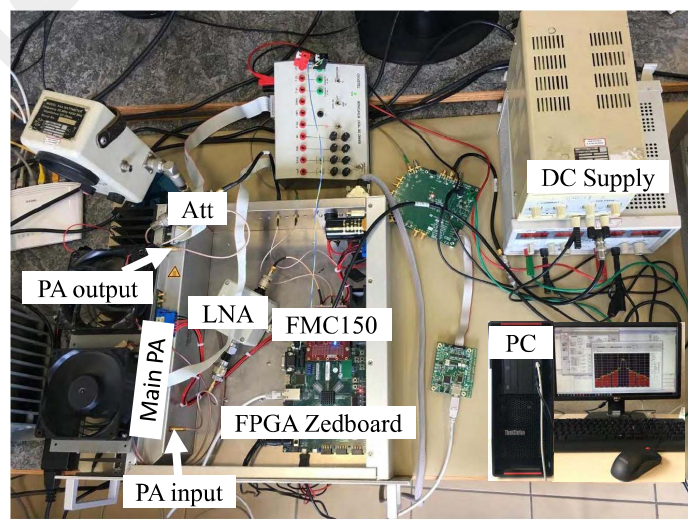

Fig. 5. Setup of measurement testbench.

\section{EXPERIMENTAL RESULTS}

\section{A. Description of Setup and Measurements}

To evaluate the efficiency of the proposed adaptive DPD technique, some measurements are made on the testbench shown in Fig. 5. High power amplifiers AMPV fabricated by Telerad, are used for our targeted applications, such as the airborne VHF Digital Link (VDL) Model-2 system. On the transmit side, the baseband signal is generated by a PC and sent to a field-programmable gate array (FPGA) Zedboard for digital signal processing (DSP) and frequency up-conversion. An RF board (FMC150) converts the signal after FPGA into the analog domain. The signal is then sent to a low noise amplifier (LNA) and finally fed into the main PA. On the receive side, the feedback loop, consisting of an 


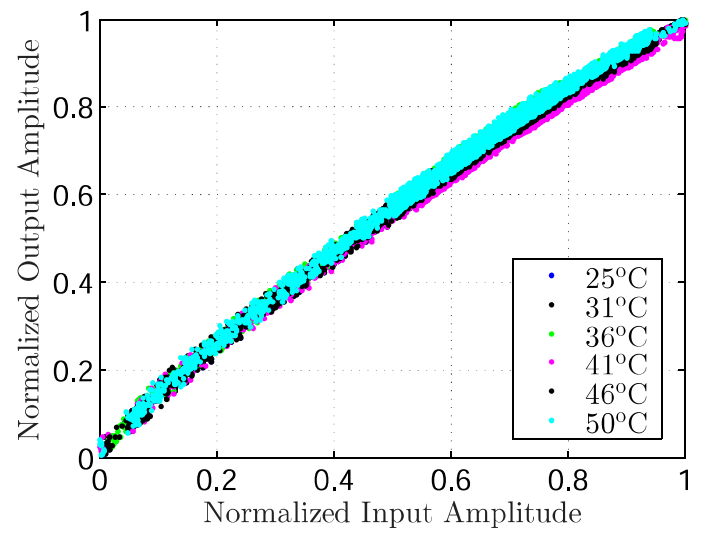

Fig. 6. Measured AM/AM characteristics of the PA at various temperatures.

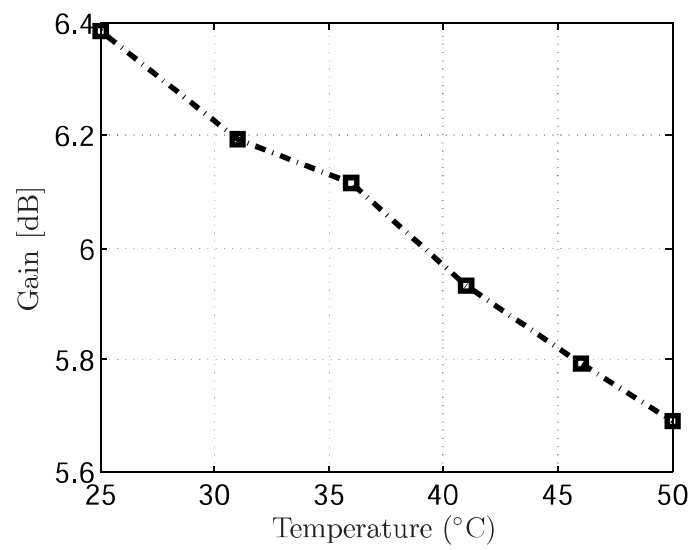

Fig. 7. Measured gain of the PA at various temperatures.

attenuator (Att), the FMC150, and FPGA Zedboard, is used to capture the PA output signal, down-convert it to baseband signal, which is then sent to a PC from FPGA Zedboard for model extraction and DPD design.

The measured input signal is a quadrature phase shift keying (QPSK) modulated signal with $16.8 \mathrm{kHz}$ bandwidth at carrier frequency of $118 \mathrm{MHz}$. The measured output power is $75 \mathrm{~W}$. Let us denote $\mathrm{ACPR}_{\mathrm{U} 1}$ and $\mathrm{ACPR}_{\mathrm{U} 2}$ as the ACPR values measured at the first and second upper adjacent channels, corresponding to frequency offsets of $25 \mathrm{kHz}$ and $50 \mathrm{kHz}$, respectively. Our targeted applications impose strong constraints on ACPRs $\left(\mathrm{ACPR}_{\mathrm{U} 1}<-65 \mathrm{~dB}\right.$ and $\left.\mathrm{ACPR}_{\mathrm{U} 1}<-75 \mathrm{~dB}\right)$ and EVM $<6 \%$. Accurate timedelay alignment between the input and output samples is executed using the cross-correlation technique [30]. After a time-alignment process, the obtained data are used to compute the instantaneous complex gain of the PA. The measured $\mathrm{AM} / \mathrm{AM}$ characteristics and gain of the PA are shown in Figs. 6 and 7, respectively, for various temperatures. From these figures, one can observe that the AM/AM characteristics and gain curve are altered as the measured temperature is varied. The gain of the PA decreases as the temperature increases. Thus, a new model of the PA should be re-extracted and used for the corresponding PD identification for each different temperature. This process makes the DPD system too cumbersome and complex. In order to linearize the PA

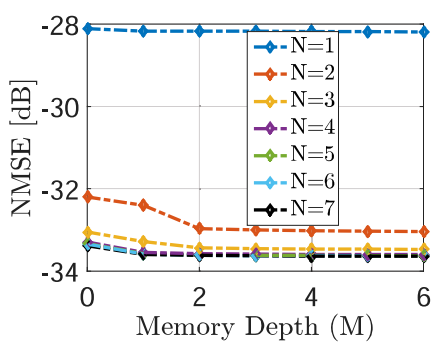

(a)

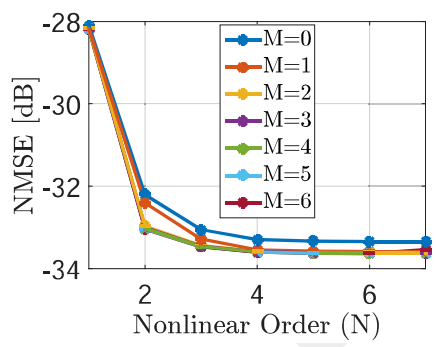

(b)
Fig. 8. NMSE performance vs. nonlinear order $(N)$ and memory depth $(M)$ measured at $50^{\circ} \mathrm{C}$. (a) NMSE vs. $M$. (b) NMSE vs. $N$.

more efficiently, the adaptive DPD is instead required to track the coefficient fluctuation of the PA.

\section{B. MP-Based Model Optimization}

Due to its low computational cost, satisfactory accuracy, and easy hardware implementation [1], [2], [14], [24], MP-based models have been widely applied for behavioral modeling and predistortion of PAs exhibiting nonlinear memory effects. Since the PA output in a real measurement is limited to the frequency band of interest, most published DPD techniques [2]-[4], [8], [14], [21] consider only odd-order terms of the polynomial. However, it is worth noting that all the terms in the polynomial, including both odd- and even-order terms for the models, should be used to achieve the best representation of the PA magnitude and phase responses [31]. Moreover, if taking only odd-order terms for the models, the required nonlinear order may be higher in order to better model the nonlinear behavior of the PA.

In this framework, we thus use the MP-based model including both odd- and even-order terms. After capturing a particular set of input and output samples measured at different temperatures, each MP-based model of the PA is determined using the LS method [3]. In order to reduce the computational complexity, the orders $(N$ and $M)$ of the MP-based models are determined using a performance-based sweeping method. Obviously, the nonlinear order and memory depth parameters affect the normalized mean square error (NMSE) and ACPR performance [32]. We additionally define ACPR deviation, based on which, the optimal orders of the models are determined. The ACPR deviation $\sigma$ is the normalized difference between ACPRs of the MP-based model output and PA output, expressed as

$$
\sigma(\%)=\left|\frac{\mathrm{ACPR}_{\text {model }}-\mathrm{ACPR}_{\mathrm{DUT}}}{\mathrm{ACPR}_{\mathrm{DUT}}}\right| \times 100
$$

where $\mathrm{ACPR}_{\text {model }}$ and $A C P R_{\mathrm{DUT}}$ are the ACPRs of the model output and the device under test (DUT) PA output, respectively. Designations $\sigma_{\mathrm{U} 1}$ and $\sigma_{\mathrm{U} 2}$ will be used to denote the measured $\sigma$ in the first and second upper adjacent channels, respectively. The NMSE and ACPR deviation of each model are evaluated as a function of the nonlinear order and memory depth over a wide range of nonlinear orders from 1 to 7 and memory depths from 0 to 6 . The results reported in Figs. 8 and 9 show that the optimal values of $M$ and $N$, 


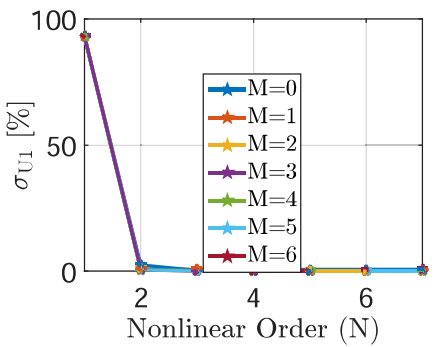

(a)

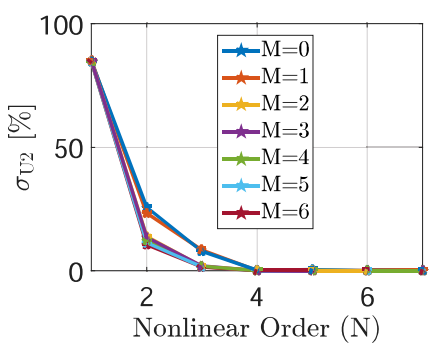

(b)
Fig. 9. ACPR deviation $\sigma$ vs. nonlinear order and memory depth with PA at $50^{\circ} \mathrm{C}$. (a) $\sigma_{\mathrm{U} 1}$. (b) $\sigma_{\mathrm{U} 2}$.

TABLE II

Performance of the Optimal MP-BAsed MODELS AT DIFFERENT TEMPERATURES

\begin{tabular}{|c|c|c|c|c|c|c|c|c|}
\hline \multicolumn{3}{|c|}{ Temperature } & $25^{\circ} \mathrm{C}$ & $31^{\circ} \mathrm{C}$ & $36^{\circ} \mathrm{C}$ & $41^{\circ} \mathrm{C}$ & $46^{\circ} \mathrm{C}$ & $50^{\circ} \mathrm{C}$ \\
\hline PA & $\begin{array}{l}\text { Input } \\
\text { Output }\end{array}$ & $\begin{array}{l}\text { ACPR-U1 } \\
\text { ACPR-U2 } \\
\text { ACPR-U1 } \\
\text { ACPR-U2 } \\
\end{array}$ & $\begin{array}{l}-70.37 \\
-84.22 \\
-36.78 \\
-45.76\end{array}$ & $\begin{array}{l}-70.24 \\
-84.17 \\
-36.46 \\
-45.35\end{array}$ & $\begin{array}{l}-70.14 \\
-84.38 \\
-36.48 \\
-45.50\end{array}$ & $\begin{array}{l}-70.35 \\
-84.11 \\
-36.28 \\
-45.21 \\
\end{array}$ & $\begin{array}{l}-70.13 \\
-84.22 \\
-36.25 \\
-45.28\end{array}$ & $\begin{array}{l}-70.54 \\
-84.25 \\
-36.53 \\
-45.62\end{array}$ \\
\hline \multirow[t]{2}{*}{ MP-based model } & Output & $\begin{array}{l}\text { ACPR-U1 } \\
\text { ACPR-U2 }\end{array}$ & $\begin{array}{l}-36.99 \\
-45.50 \\
\end{array}$ & $\begin{array}{l}-36.68 \\
-45.11 \\
\end{array}$ & $\begin{array}{l}-36.72 \\
-45.30 \\
\end{array}$ & $\begin{array}{l}-36.51 \\
-45.00 \\
\end{array}$ & $\begin{array}{l}-36.48 \\
-45.09 \\
\end{array}$ & $\begin{array}{l}-36.77 \\
-45.40 \\
\end{array}$ \\
\hline & & $\begin{array}{l} \\
\text { L } \\
\text { Coefs }\end{array}$ & $\begin{array}{c}5 \\
1 \\
10\end{array}$ & $\begin{array}{c}5 \\
1 \\
10\end{array}$ & $\begin{array}{c}5 \\
1 \\
10\end{array}$ & $\begin{array}{c}5 \\
1 \\
10\end{array}$ & $\begin{array}{c}5 \\
1 \\
10\end{array}$ & $\begin{array}{c}5 \\
1 \\
10\end{array}$ \\
\hline
\end{tabular}

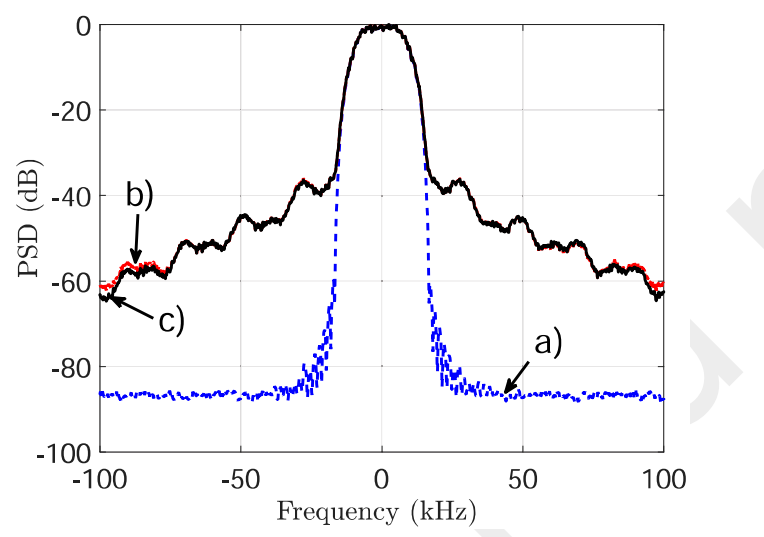

Fig. 10. Power spectral density (PSD) at $50^{\circ} \mathrm{C}$ : a) PA input, b) PA output, c) MP-based model output.

to achieve the best NMSE and ACPR deviation, are 1 and 5, respectively. Analogously, the above mentioned performancebased sweeping method is applied to determine optimal models for different temperatures. As can be seen from Table II (bottom three rows), the optimal values of the nonlinear order and memory depth remain constant for all models when the temperature changes. The MP-based models exactly describe the nonlinear behavior of the PA, i.e., the ACPR values of the MP model output are identical to those of the PA output. As a result, the output spectrum of the model coincides with that of the PA, as shown in Fig. 10.

Fig. 11 shows the coefficient variation of the optimal MPbased models, which all have the same optimal nonlinear order and memory depth. It is clear that the values of their coefficients change in a relatively smooth way over temperature. Therefore, the proposed additional ILA-based DPD can easily follow these variations.

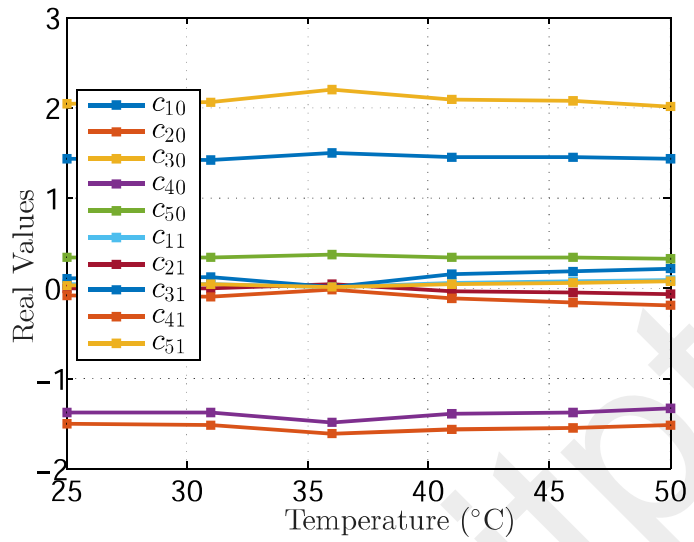

(a)

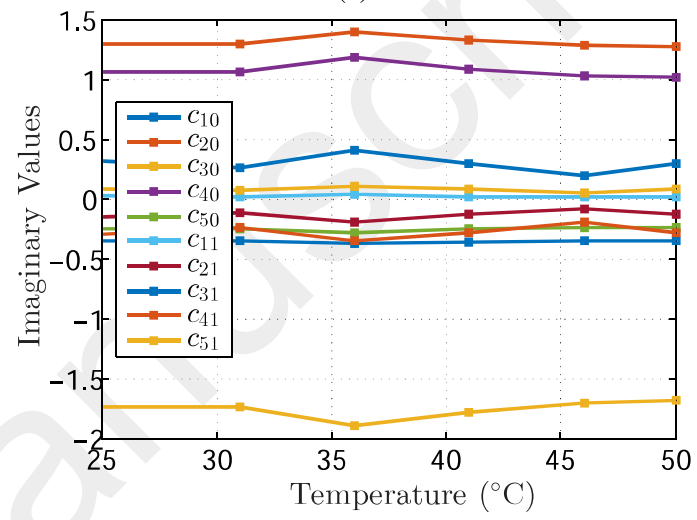

(b)

Fig. 11. Coefficient variation of the optimal MP-based models. (a) Real part. (b) Imaginary part.

\section{Validation of Proposed Digital Predistortion}

Without loss of generality, the offline LILUT-DLA-based $\mathrm{PD}$ designed for the PA model at $25^{\circ} \mathrm{C}$ can be used to compensate the nonlinear behavior of the PA at various temperatures. The coefficients of the optimal PA models are first extracted using the LS technique with data measured from the input and output of the PA at multiple temperatures. We test various DPDs, including offline LILUT-DLA-based DPD, offline LS-ILA-based DPD, adaptive LMS- or RLS-ILA-based DPD, and our proposed DPD, when the temperature changes.

As presented in Table II, the optimal values of the nonlinear order and memory depth of the PA model are selected as 5 and 1, respectively. In the stand-alone DLA-based DPD, we also select $N=5$ and $M=1$. The LUT size is chosen by simulation to be 32 in order to achieve a good trade-off between performance and complexity [32].

In our approach (Design+LMS and Design+RLS), the initial weight vector is chosen such that the first element is 1 and the others are zero, i.e., $\hat{\omega}(0)=[1,0, \cdots, 0]^{\mathrm{T}}$. In Design+LMS, the step-size $\mu$ is chosen to be 0.001 in order to obtain a good compromise between convergence speed and parameter estimation precision [29]. In Design+RLS, the initial inverse correlation matrix is a diagonal matrix whose diagonal elements are set at $10^{6}$. The forgetting factor $\lambda$ is selected as 0.995 . After the orders of the PA model 


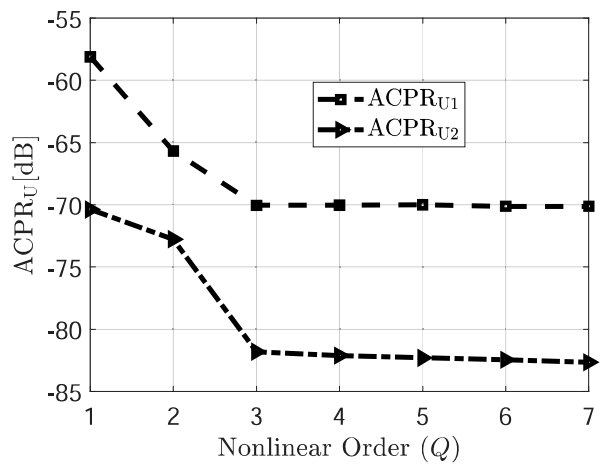

Fig. 12. ACPR vs. $Q$ for $P=1$.

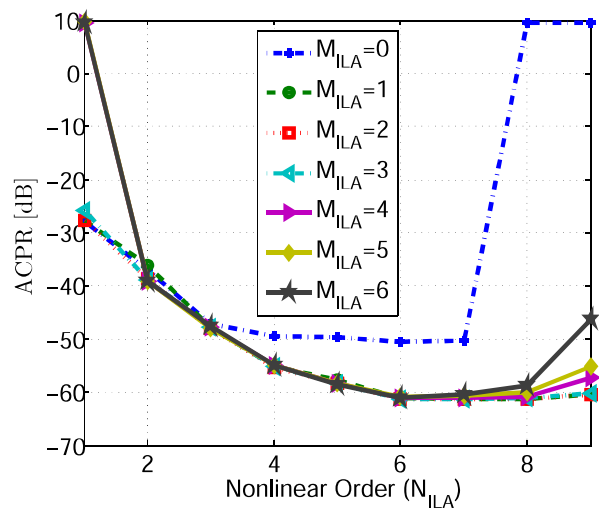

Fig. 13. ACPR vs. $N_{\mathrm{ILA}}$ for different values of $M_{\mathrm{ILA}}$.

and DLA-based PD model have been optimized, the orders $(Q, P)$ of the proposed ILA-based PD model are analogously optimized based on the ACPR performance. ACPR values versus $Q$ are shown in Fig. 12 for $P=1$. Obviously, the optimal value of $Q$ is 3 , at which $\mathrm{ACPR}_{\mathrm{U} 1}$ and $\mathrm{ACPR}_{\mathrm{U} 2}$ are equal to $-70 \mathrm{~dB}$ and $-82 \mathrm{~dB}$, respectively. These ACPR values are almost equal to those of the PA input.

In the adaptive stand-alone ILA-based DPD, the initial weight vector, step-size, and initial inverse correlation matrix are chosen to be the same as those in our approach. The nonlinear order $N_{\text {ILA }}$ and memory depth $M_{\text {ILA }}$ of the MP-based models of both PD and training block are optimally selected as 6 and 1, respectively, in order to achieve the minimum ACPR, as shown in Fig. 13.

We first investigate the performance of the offline LILUT-DLA-based DPD presented in Section II and the offline LS-ILA-based DPD proposed in [3], using a model of a PA operating at $25^{\circ} \mathrm{C}$. We observe a significant reduction in spectral regrowth for both linearizion techniques, as shown in Fig. 14. However, the offline DLA-based PD suppresses most of the spectral regrowth and shows better performance than the offline ILA-based PD. This is due to the fact that the measurement noise appears at the PA output and the ILAbased DPD is more sensitive to noise than the DLA-based DPD [12].

We next investigate the performance of various DPDs, including the offline LILUT-DLA-based DPD and the proposed adaptive DPD, when the temperature changes.

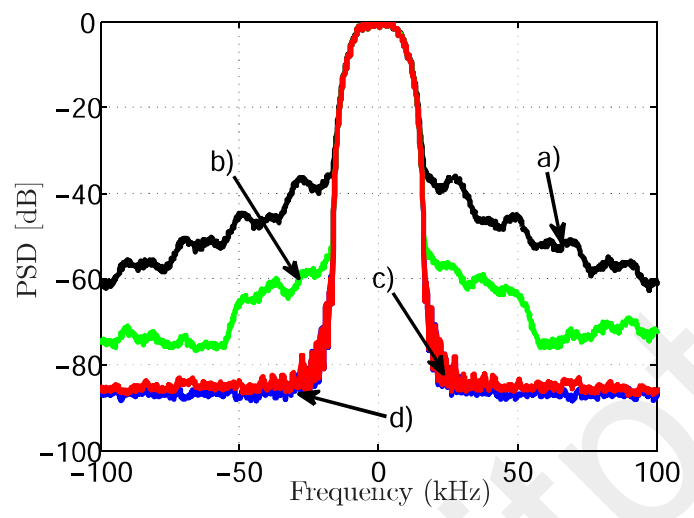

Fig. 14. Effectiveness of offline DPDs in suppressing spectral regrowth for the PA model at $25^{\circ} \mathrm{C}$ : a) output, b) offline stand-alone LS-ILA-based DPD, c) offline stand-alone LILUT-DLA-based DPD, d) input.

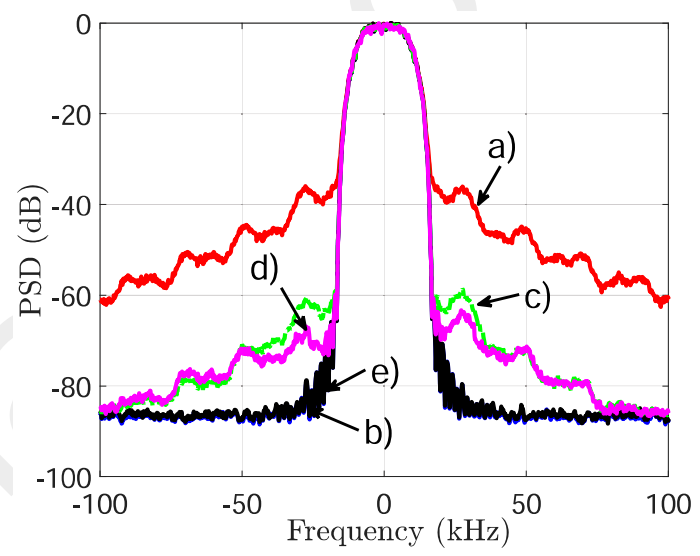

Fig. 15. Effectiveness of various DPDs in suppressing spectral regrowth for the PA at $50^{\circ} \mathrm{C}$ : a) PA Output, b) PA Input, c) Offline LILUT-DLA-based DPD, d) Design+LMS, e) Design+RLS.

Fig. 15 shows the obtained results, for instance, at $50^{\circ} \mathrm{C}$. Obviously, the offline LILUT-DLA-based DPD does not completely suppress the spectral regrowth because the PA characteristics change with temperature drift. A further reduction in the residual spectral regrowth can be achieved by Design+LMS because the adaptation loop updates the coefficients of the ILA-based PD to compensate the residual spectral regrowth. Design+RLS outperforms the others. It can fully mitigate the spectral regrowth due to the major advantages of the RLS algorithm in obtaining faster convergence and lower mean-square error, as clearly shown in Fig. 16. The RLS algorithm converges in about 1700 samples, while the LMS algorithm has not fully converged after about 5000 samples.

As presented above, some residual nonlinear distortion will appear after executing the offline LILUT-DLA-based PD when the PA characteristics change due to the temperature drift. As a result, the static DLA-based DPD obtains around $-60 \mathrm{~dB}$ and $-70 \mathrm{~dB}$ of $\mathrm{ACPR}_{\mathrm{U} 1}$ and $\mathrm{ACPR}_{\mathrm{U} 2}$, respectively over temperature variation, as shown in Fig. 17, which does not satisfy the ACPR requirements. In other words, it can not effectively follow changes in the PA characteristics. In contrast, by employing the proposed approach, the distortion of the PA at each temperature can be effectively corrected. 


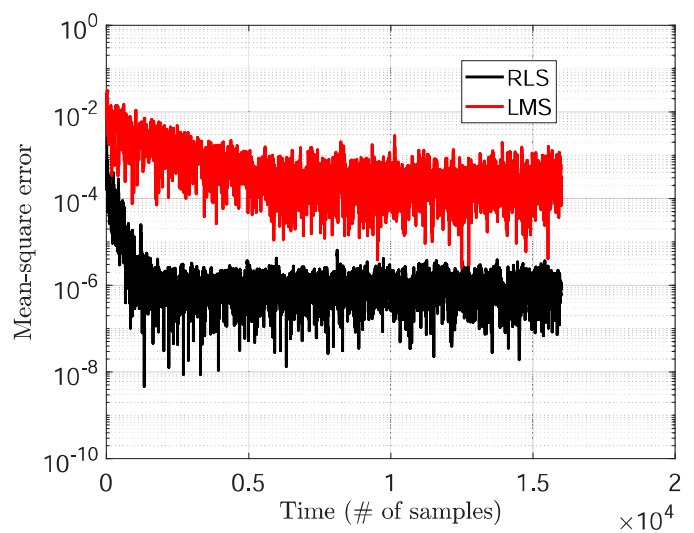

Fig. 16. Learning curves for Design+LMS and Design+RLS.

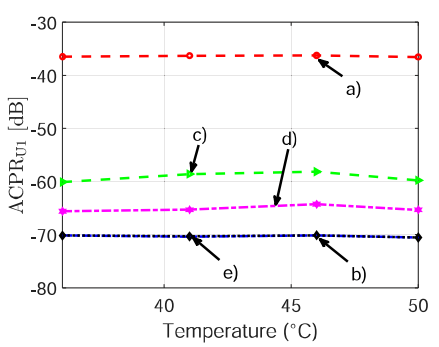

(a)

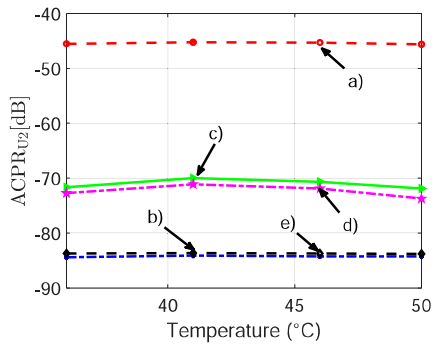

(b)
Fig. 17. ACPR performance when PA temperature changes: a) PA output, b) Input, c) Offline LILUT-DLA-based DPD, d) Design+LMS, and e) Design+RLS. (a) ACPR $\mathrm{U}_{1}$. (b) $\mathrm{ACPR}_{\mathrm{U} 2}$.

TABLE III

EVM PERFORMANCE OF OUR PROPOSED DESIGN+RLS

\begin{tabular}{l|c|c|c|c|c}
\hline Temperature $\left({ }^{\circ} \mathrm{C}\right)$ & 31 & 36 & 41 & 46 & 50 \\
\hline EVM $(\%)$ & 0.49 & 0.52 & 0.54 & 0.40 & 0.44 \\
\hline
\end{tabular}

The proposed Design+RLS achieves $-70 \mathrm{~dB}$ and $-83 \mathrm{~dB}$ of $\mathrm{ACPR}_{\mathrm{U} 1}$ and $\mathrm{ACPR}_{\mathrm{U} 2}$, respectively, which are almost equal to the ACPR values of the input signal. Therefore, the ACPR requirements are fully satisfied. Moreover, the obtained EVM values are always less than $1 \%$, as presented in Table III. This shows that the proposed solution is able to accurately follow changes in the PA characteristics due to temperature shift.

In order to show that the proposed DPD is more robust than the stand-alone ILA-based DPD with respect to the noise at the PA output, we analyze the condition number of the correlation matrix of the output samples of both the CDPA in the proposed DPD and the tested PA in the stand-alone LS-ILA-based DPD. Fig. 18 shows the condition number of these two approaches. Thanks to the incorporation of DLA, the CDPA is much more linear than the tested PA, leading to a lower condition number of its correlation matrix than that of the PA in the stand-alone ILA-based DPD. This indicates that the proposed architecture is more robust than the ILA-based DPD with respect to the measurement noise.

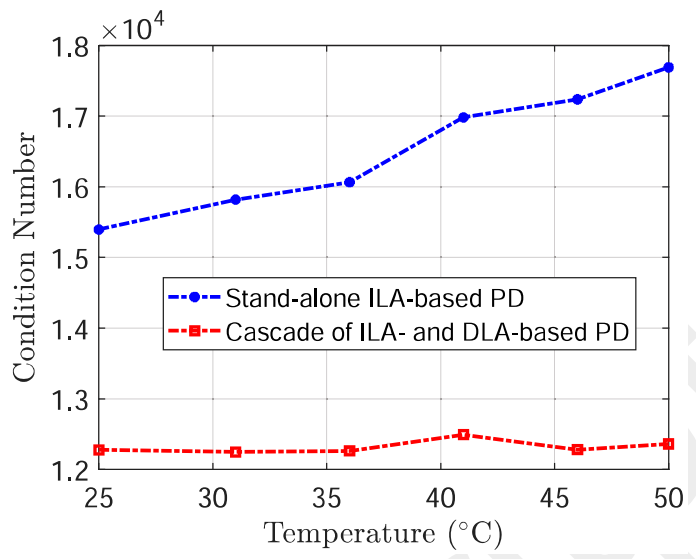

Fig. 18. Condition number at various temperatures.

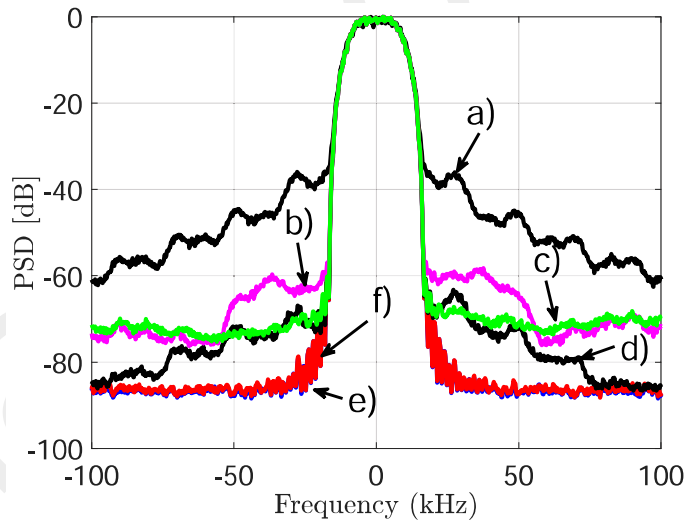

Fig. 19. Effectiveness of adaptive DPDs in suppressing spectral regrowth: a) PA output, b) stand-alone LMS-ILA-based DPD, c) stand-alone RLS-ILAbased DPD, d) Design+LMS, e) Design+RLS, f) PA input.

\section{Comparison of Proposed Adaptive DPD With the Stand-Alone LMS-ILA- and RLS-ILA-Based DPDs}

This section makes a comparison of the effectiveness of predistortion in suppressing spectral regrowth between the proposed adaptive DPD and adaptive stand-alone ILA-based DPD. Fig. 19 shows an efficiency comparison in canceling the spectral regrowth at $50^{\circ} \mathrm{C}$. Obviously, the stand-alone RLSILA-based DPD shows better spectral regrowth suppressing than the LMS-ILA-based DPD. Neither of the stand-alone ILA-based DPDs is able to completely suppress the spectral regrowth due to measurement noise at the PA output. Noisy measurements seriously influence the efficiency of the ILAbased DPD [12], [17], [33]. The stand-alone RLS-ILA-based DPD has performance in reducing the spectral regrowth as good as the proposed Design+LMS. As previously presented, the proposed DPD architecture is more robust than the stand-alone ILA-based DPD with respect to the PA output noise. Moreover, thanks to the smaller mean-square error of RLS, the proposed Design+RLS almost fully reduces the out-of-band distortion to the noise floor. As a result, it effectively compensates the PA distortion at each temperature, obtaining $-70 \mathrm{~dB}$ and $-83 \mathrm{~dB}$ of $\mathrm{ACPR}_{\mathrm{U} 1}$ and $\mathrm{ACPR}_{\mathrm{U} 2}$, respectively, which are almost equal to the ACPRs 


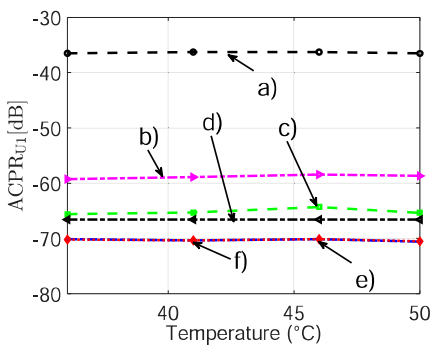

(a)

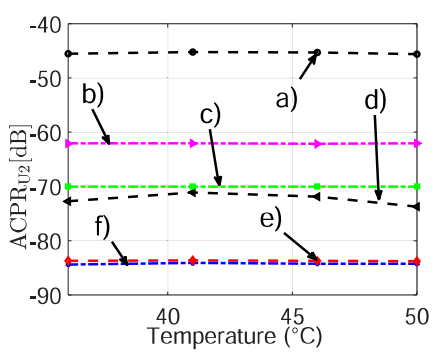

(b)
Fig. 20. ACPR performance of various adaptive DPDs: a) PA output, b) stand-alone LMS-ILA-based DPD, c) stand-alone RLS-ILA-based DPD, d) Design+LMS, e) Design+RLS, and f) PA input. (a) $\mathrm{ACPR}_{\mathrm{U} 1}$. (b) $\mathrm{ACPR}_{\mathrm{U} 2}$.

of the input signal and better than the ACPRs obtained by the stand-alone ILA-based DPD, as shown in Fig. 20. It also uses a smaller number of adaptive coefficients than the adaptive stand-alone ILA-based DPD, which makes the proposed approach converge more quickly. In other words, the proposed Design+RLS significantly outperforms the other compared techniques. It is worth pointing out that although the experimental results for narrowband signals are presented, we have checked the applicability of the proposed method to wideband signals/PAs by simulation using a Wiener model for the PA. The obtained results show that the proposed method has excellent performance in linearizing wideband PAs with significant nonlinear memory effects. As a result, the proposed approach should be applicable for wideband PA linearization.

\section{CONCLUSION}

In this paper, a novel adaptive DPD architecture has been proposed in order to track nonlinear behavior changes in PA characteristics. The proposed architecture is constructed by cascading the adaptive ILA-based PD and the static LILUT-DLA-based PD. The static LILUT-DLA-based PD is designed to linearize the PA for a specific condition such that the cascaded system of the DLA-based PD and PA, named CDPA, is theoretically linear. In real-time applications, when PA characteristics change due to PVT drifts, the CDPA is no longer linear, which causes some residual nonlinear distortion at the PA output. The proposed additional adaptive ILA-based PD placed in front of the CDPA can effectively compensate this residual nonlinear distortion. Thanks to the static DLAbased PD, most of the nonlinear memory effects of the PA are mitigated, making the CDPA much more linear than the PA. As a result, the correlation matrix of the PA output samples is better conditioned. The proposed solution is more robust to measurement noise and guarantees a better preinverse model for nonlinear PAs than the classical stand-alone ILA-based DPD. Moreover, the combined adaptive ILA-based PD in our DPD design is simpler and requires a smaller number of adaptive coefficients than the adaptive stand-alone ILA-based DPD, which makes the proposed approach converge more quickly. The measurement results confirm that the proposed Design+RLS fully compensates the spectral regrowth of the PA output even if the PA characteristics change, and obtains $-70 \mathrm{~dB}$ and $-83 \mathrm{~dB}$ of $\mathrm{ACPR}_{\mathrm{U} 1}$ and $\mathrm{ACPR}_{\mathrm{U} 2}$, respectively, which are almost equal to the ACPRs of the input signals. Consequently, It shows better linearization performance than both the stand-alone DLA- and stand-alone ILA-based DPDs.

\section{ACKNOWLEDGMENT}

APOGEES project has been labeled by pole Aerospace Valley, pole Images et Reseaux and pole Alpha-RLH in the framework of the French FUI22 research program.

\section{REFERENCES}

[1] F. M. Ghannouchi and O. Hammi, "Behavioral modeling and predistortion," IEEE Microw. Mag., vol. 10, no. 7, pp. 52-64, Dec. 2009.

[2] L. Guan and A. Zhu, "Green communications: Digital predistortion for wideband RF power amplifiers," IEEE Microw. Mag., vol. 15, no. 7, pp. 84-99, Nov. 2014.

[3] L. Ding et al., "A robust digital baseband predistorter constructed using memory polynomials," IEEE Trans. Commun., vol. 52, no. 1, pp. 159-165, Jan. 2004.

[4] J. Chani-Cahuana, P. N. Landin, C. Fager, and T. Eriksson, "Iterative learning control for RF power amplifier linearization," IEEE Trans. Microw. Theory Techn., vol. 64, no. 9, pp. 2778-2789, Sep. 2016.

[5] J. Kim, C. Park, J. Moon, and B. Kim, "Analysis of adaptive digital feedback linearization techniques," IEEE Trans. Circuits Syst. I, Reg. Papers, vol. 57, no. 2, pp. 345-354, Feb. 2010.

[6] Y. Guo, C. Yu, and A. Zhu, "Power adaptive digital predistortion for wideband RF power amplifiers with dynamic power transmission," IEEE Trans. Microw. Theory Techn., vol. 63, no. 11, pp. 3595-3607, Nov. 2015.

[7] M. Schoukens, J. Hammenecker, and A. Cooman, "Obtaining the preinverse of a power amplifier using iterative learning control," IEEE Trans. Microw. Theory Techn., vol. 65, no. 11, pp. 4266-4273, Nov. 2017.

[8] X. Feng, B. Feuvrie, A. S. Descamps, and Y. Wang, "Improved baseband digital predistortion for linearising PAs with nonlinear memory effects using linearly interpolated LUT," Electron. Lett., vol. 49, no. 22, pp. 1389-1391, Oct. 2013.

[9] D. Zhou and V. E. DeBrunner, "Novel adaptive nonlinear predistorters based on the direct learning algorithm," IEEE Trans. Signal Process., vol. 55, no. 1, pp. 120-133, Jan. 2007.

[10] R. N. Braithwaite, "A comparison of indirect learning and closed loop estimators used in digital predistortion of power amplifiers," in IEEE MTT-S Int. Microw. Symp. Dig., May 2015, pp. 1-4.

[11] S. Choi, E. R. Jeong, and Y. H. Lee, "Adaptive predistortion with direct learning based on piecewise linear approximation of amplifier nonlinearity," IEEE J. Sel. Topics Signal Process., vol. 3, no. 3, pp. 397-404, Jun. 2009.

[12] M. A. Hussein, V. A. Bohara, and O. Venard, "On the system level convergence of ILA and DLA for digital predistortion," in Proc. Int. Symp. Wireless Commun. Syst. (ISWCS), Aug. 2012, pp. 870-874.

[13] C. Eun and E. J. Powers, "A new Volterra predistorter based on the indirect learning architecture," IEEE Trans. Signal Process., vol. 45, no. 1, pp. 223-227, Jan. 1997.

[14] D. R. Morgan, Z. Ma, and L. Ding, "Reducing measurement noise effects in digital predistortion of RF power amplifiers," in Proc. IEEE Int. Conf. Commun. (ICC), vol. 4, May 2003, pp. 2436-2439.

[15] F.-L. Luo, Digital Front-End in Wireless Communications and Broadcasting: Circuits and Signal Processing. Cambridge, U.K.: Cambridge Univ. Press, 2011. [Online]. Available: http://cds.cern.ch/record/1539386

[16] P. N. Landin, A. E. Mayer, and T. Eriksson, "MILA-A noise mitigation technique for RF power amplifier linearization," in Proc. IEEE 11th Int. Multi-Conf. Syst., Signals Devices (SSD), Feb. 2014, pp. 1-4.

[17] H. Paaso and A. Mammela, "Comparison of direct learning and indirect learning predistortion architectures," in Proc. IEEE Int. Symp. Wireless Commun. Syst., Oct. 2008, pp. 309-313.

[18] Y. H. Lim, Y. S. Cho, I. W. Cha, and D. H. Youn, "An adaptive nonlinear prefilter for compensation of distortion in nonlinear systems," IEEE Trans. Signal Process., vol. 46, no. 6, pp. 1726-1730, Jun. 1998.

[19] H. W. Kang, Y. S. Cho, and D. H. Youn, "On compensating nonlinear distortions of an OFDM system using an efficient adaptive predistorter," IEEE Trans. Commun., vol. 47, no. 4, pp. 522-526, Apr. 1999.

[20] R. N. Braithwaite, "Reducing estimator biases due to equalization errors in adaptive digital predistortion systems for RF power amplifiers," in IEEE MTT-S Int. Microw. Symp. Dig., Jun. 2012, pp. 1-3. 
[21] P. M. Suryasarman and A. Springer, "A comparative analysis of adaptive digital predistortion algorithms for multiple antenna transmitters," IEEE Trans. Circuits Syst. I, Reg. Papers, vol. 62, no. 5, pp. 1412-1420, May 2015.

[22] X. Feng, B. Feuvrie, A. S. Descamps, and Y. Wang, "A digital predistortion method based on nonuniform memory polynomial model using interpolated LUT," in Proc. IEEE Top. Conf. Power Amplif. Wireless Radio Appl. (PAWR), Jan. 2015, pp. 1-3.

[23] J. Kim and K. Konstantinou, "Digital predistortion of wideband signals based on power amplifier model with memory," Electron. Lett., vol. 37, no. 23, pp. 1417-1418, Nov. 2001.

[24] A. E. Abdelrahman, O. Hammi, A. K. Kwan, A. Zerguine, and F. M. Ghannouchi, "A novel weighted memory polynomial for behavioral modeling and digital predistortion of nonlinear wireless transmitters," IEEE Trans. Ind. Electron., vol. 63, no. 3, pp. 1745-1753, Mar. 2016.

[25] E. Cottais, Y. Wang, and S. Toutain, "A new adaptive baseband digital predistortion technique," in Proc. Eur. Microw. Assoc., vol. 2, Jun. 2006 , pp. $154-159$.

[26] F. Li, B. Feuvrie, Y. Wang, and W. Chen, "MP/LUT baseband digital predistorter for wideband linearisation," Electron. Lett., vol. 47, no. 19, pp. 1096-1098, Sep. 2011.

[27] A. Hekkala et al., "Predistortion of radio over fiber links: Algorithms, implementation, and measurements," IEEE Trans. Circuits Syst. I, Reg. Papers, vol. 59, no. 3, pp. 664-672, Mar. 2012.

[28] A. V. Oppenheim and R. W. Schafer, Digital Signal Processing. Upper Saddle River, NJ, USA: Prentice-Hall, 2006.

[29] S. Haykin, Adaptive Filter Theory. Upper Saddle River, NJ, USA Prentice-Hall, 2002.

[30] T. Liu, S. Boumaiza, and F. M. Ghannouchi, "Deembedding static nonlinearities and accurately identifying and modeling memory effects in wide-band RF transmitters," IEEE Trans. Microw. Theory Techn., vol. 53, no. 11, pp. 3578-3587, Nov. 2005.

[31] L. Ding and G. T. Zhou, "Effects of even-order nonlinear terms on power amplifier modeling and predistortion linearization," IEEE Trans. Veh. Technol., vol. 53, no. 1, pp. 156-162, Jan. 2004

[32] X. Feng, "Efficient baseband digital predistortion techniques for linearizing power amplifier by taking into account nonlinear memory effect," Ph.D. dissertation, Univ. Nantes, Nantes, France, Sep. 2015. [Online]. Available: https://hal.archives-ouvertes.fr/tel-01206266

[33] X. Feng, Y. Wang, B. Feuvrie, A.-S. Descamps, Y. Ding, and Z. Yu, "Analysis on LUT based digital predistortion using direct learning architecture for linearizing power amplifiers," EURASIP J. Wireless Commun. Netw., vol. 2016, no. 1, p. 132, Dec. 2016. [Online]. Available: https://doi.org/10.1186/s13638-016-0628-y 